\title{
Percentile curves for cardiometabolic disease markers in Canadian children and youth: a cross-sectional study
}

\author{
Nicole Ata', Bryan Maguire ${ }^{2}$, David C Hamilton ${ }^{1}$ and Stefan Kuhle ${ }^{1 *}$ (D)
}

\begin{abstract}
Background: The objective of this study to develop percentile curves for cardiometabolic disease markers in a population-based sample of Canadian children and youth.

Methods: The analysis used data from 6116 children and adolescents between 6 and 19 years of age who participated in the Canadian Health Measures Survey cycles 1 (2007/2009), 2 (2009/2011), and 3 (2012/2013). Total cholesterol, HDL cholesterol, and hemoglobin A1c levels as well as fasting levels of triglycerides, insulin, and homeostasis model assessment insulin resistance were measured using standardized procedures. Age- and sex-specific centiles for all markers were calculated using Cole and Green's LMS method.
\end{abstract}

Results: With the exception of hemoglobin A1c, all markers showed age- and sex-related differences during childhood and adolescence.

Conclusions: We have developed centile curves for cardiometabolic disease markers in Canadian children and adolescents and demonstrated age and sex differences that should be considered when evaluating these markers in this age group.

Keywords: Child, Adolescent, Metabolism, Obesity, Development, Diabetes

\section{Background}

Cardiovascular disease (CVD) is currently the leading cause of death worldwide [1]. With the exception of congenital heart disease, CVD manifests in adulthood, but its risk factors are already detectable in childhood. Abnormal blood lipids and diabetes are among the risk factors for the development of CVD [2,3]. An abnormal lipid profile can include elevated total cholesterol, elevated triglycerides, and low high-density lipoprotein (HDL) cholesterol. Insulin resistance plays an important role in the development of youth-onset type 2 diabetes, an emerging disease in children and youth [4]. Homeostasis model assessment estimates insulin resistance (HOMA-IR) from fasting levels of insulin and glucose [5]; other measures that have been used to identify insulin resistance or diabetes include fasting insulin and glycosylated hemoglobin (HbA1c), respectively [6-8].

\footnotetext{
* Correspondence: stefan.kuhle@dal.ca

${ }^{1}$ Departments of Pediatrics and Obstetrics \& Gynaecology, Dalhousie

University, Halifax, NS, Canada

Full list of author information is available at the end of the article
}

Levels of these markers vary by sex and across age in childhood and adolescence, and percentile curves have been developed to describe their physiologic development. Percentile curves have been published for lipids and markers of insulin resistance in various populations [9-14]. Since these curves are specific to populations and there are no percentile curves for the levels of these markers in Canadian children, the objective of this study was to develop percentile curves for cardiometabolic markers in a population-based sample of Canadian children and youth.

\section{Methods}

\section{Study design}

This study used data of children and youth aged 6 to 19 years from the Canadian Health Measures Survey (CHMS) cycles 1 to 3 , a representative, cross-sectional survey assessing health and wellness in Canadians [1517]. The survey includes a household interview to

(c) The Author(s). 2018 Open Access This article is distributed under the terms of the Creative Commons Attribution 4.0 International License (http://creativecommons.org/licenses/by/4.0/), which permits unrestricted use, distribution, and reproduction in any medium, provided you give appropriate credit to the original author(s) and the source, provide a link to the Creative Commons license, and indicate if changes were made. The Creative Commons Public Domain Dedication waiver (http://creativecommons.org/publicdomain/zero/1.0/) applies to the data made available in this article, unless otherwise stated. 
obtain sociodemographic and health information and a visit to a mobile examination centre to perform physical measurements and tests. The sampling frame of the Canadian Labour Force Survey was used to identify the collection sites for the mobile examination centres. Within each collection site, households were selected using the 2006 Census as the sampling frame. Interviews and examinations for the CHMS Cycle 1 were performed between 2007 and 2009, for Cycle 2 between 2009 and 2011, and for Cycle 3 between 2012 and 2013. Household response rates were $69.6,75.9$, and $74.1 \%$, respectively; final response rates in the 3 cycles were 51.7 , 55.7 and $51.7 \%$, respectively [15-17]. We combined data from the 3 cycles as per Statistics Canada guidelines [18]. A total of 11,999 persons participated in physical examination part of the three survey cycles. The present analysis uses data from 6116 children and adolescents between 6 and 19 years of age.

The Health Canada Research Ethics Board gave approval for the CHMS. All participants gave written informed consent; parents or guardians consented on behalf of children aged 6 to 13 years, and the child provided their assent to participate; youth 14 to 17 years consented on their own, but their parents or guardians had to give verbal permission for the household interview [15]. The current project was approved by the IWK Health Centre Research Ethics Board, Halifax, NS, Canada (File \# 1014413).

\section{Laboratory measurements}

Blood for measurement of cardiometabolic markers was collected by standard venipuncture. Fasted blood samples for measurement of insulin, glucose, and triglycerides were taken in a randomly selected sample of participants. The sample was obtained by randomly offering each respondent a clinic appointment either in the morning (after an overnight fast) or in the afternoon (non-fasted) [15]. Blood samples were centrifuged within $2 \mathrm{~h}$ and aliquoted within $4 \mathrm{~h}$ of collection. The samples were stored either in the refrigerator or in the freezer until shipping. Samples were shipped once a week to the Health Canada reference laboratory in Ottawa. Participants with diabetes were excluded from the analysis of insulin, HOMA-IR, and HbA1c; participants taking lipid-lowering medication were excluded from the analysis of lipids. Levels of total cholesterol, HDL cholesterol, triglycerides, and glucose were measured using a colorimetric test and $\mathrm{HbA1c}$ was measured using a immunoturbidimetric test on the Vitros 5,1FS (Ortho Clinical Diagnostics, Markham, ON, Canada). Fasting insulin levels were determined using a solid-phase, two-site chemiluminescent immunometric assay on the Advia Centaur XP (Siemens, Erlangen, Germany). Since insulin measurements in cycle 1 were performed using a different method and had a considerable proportion of levels below the test's limit of detection, we only used insulin measurements from cycles 2 and 3 in the present analysis. Fasting insulin and glucose levels were used to calculate HOMA-IR as (fasting insulin $[\mu \mathrm{U} / \mathrm{L}] \mathrm{x}$ glucose $[\mathrm{mmol} / \mathrm{L}]) / 22.5[19]$.

\section{Statistical analysis}

Percentile curves for total cholesterol, HDL cholesterol, triglycerides, insulin, HOMA-IR, and HbA1c were modeled using the LMS method by Cole and Green [20]. We have described the LMS method in

Table 1 Characteristics of 6116 Canadian children and youth aged 6 to 19 years in the Canadian Health Measures Survey Cycles 1 to 3

\begin{tabular}{|c|c|}
\hline & Prevalence [\%] \\
\hline \multicolumn{2}{|l|}{ Sex } \\
\hline Male & 51.6 \\
\hline Female & 48.4 \\
\hline \multicolumn{2}{|l|}{ Region of Canada } \\
\hline Atlantic Canada & 6.7 \\
\hline Québec & 22.5 \\
\hline Ontario & 40.5 \\
\hline Prairies & 18.2 \\
\hline British Columbia & 12.2 \\
\hline \multicolumn{2}{|l|}{ Racial origin } \\
\hline White & 80.9 \\
\hline Black & $5.9^{\mathrm{a}}$ \\
\hline Asian & $10.7^{\mathrm{a}}$ \\
\hline Other & $2.4^{\mathrm{a}}$ \\
\hline \multicolumn{2}{|l|}{ Weight status (IOTF) } \\
\hline Underweight & 7.5 \\
\hline Normal weight & 65.6 \\
\hline Overweight & 17.4 \\
\hline Obese & 9.5 \\
\hline \multicolumn{2}{|l|}{ Household education } \\
\hline Secondary school or less & 15.7 \\
\hline College & 46.6 \\
\hline University & 37.6 \\
\hline \multicolumn{2}{|l|}{ Household income } \\
\hline$\$ 30,000$ or less & 15.1 \\
\hline$\$ 30,001-\$ 60,000$ & 22.8 \\
\hline$\$ 60,001-\$ 80,000$ & 18.3 \\
\hline$\$ 80,001-\$ 100,000$ & 16.3 \\
\hline$>\$ 100,000$ & 27.5 \\
\hline
\end{tabular}

Abbreviations: IOTF International Obesity Task Force ${ }^{a}$ Coefficient of variation between 16.6 and 33.3\%; interpret with caution as per Statistics Canada sampling variability reporting guidelines 

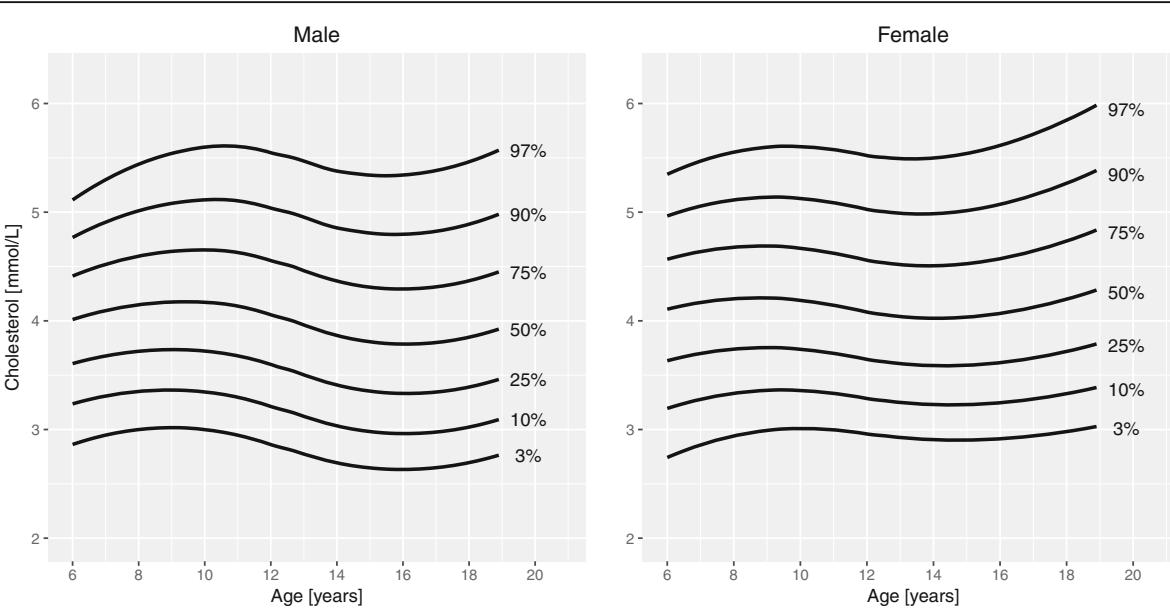

Fig. 1 Percentile curves for total cholesterol levels for male and female Canadian children and youth aged 6 to 19 years
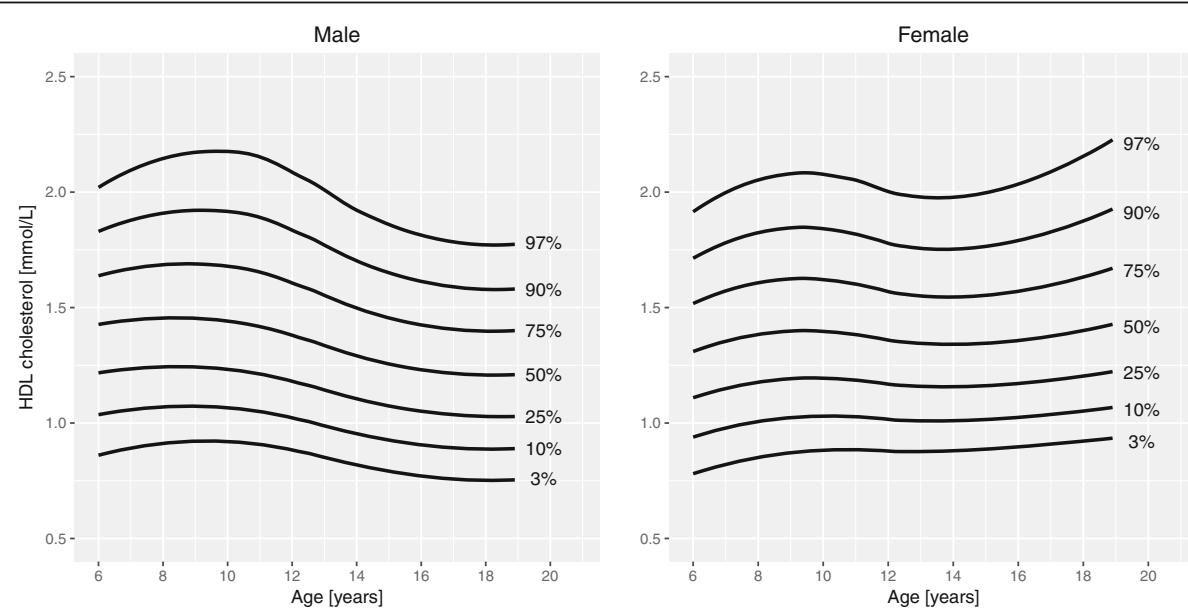

Fig. 2 Percentile curves for high-density lipoprotein (HDL) cholesterol levels for male and female Canadian children and youth aged 6 to 19 years
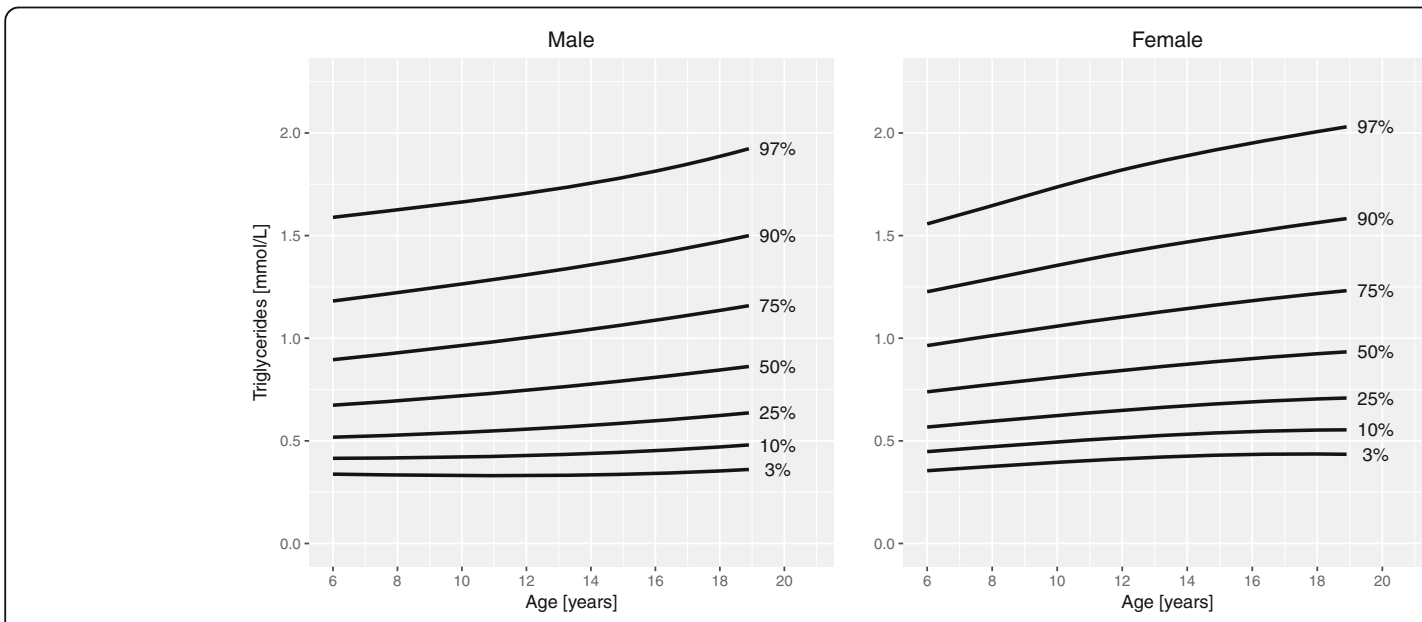

Fig. 3 Percentile curves for triglyceride levels for male and female Canadian children and youth aged 6 to 19 years 

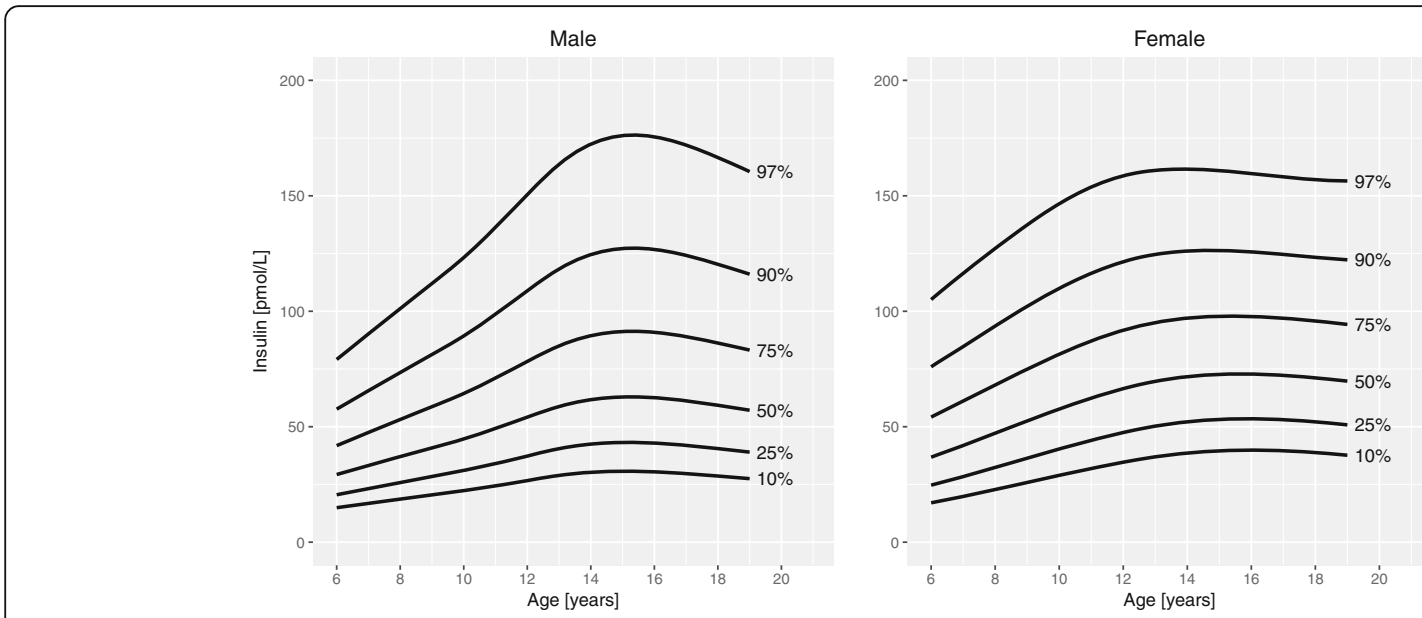

Fig. 4 Percentile curves for insulin levels for male and female Canadian children and youth aged 6 to 19 years
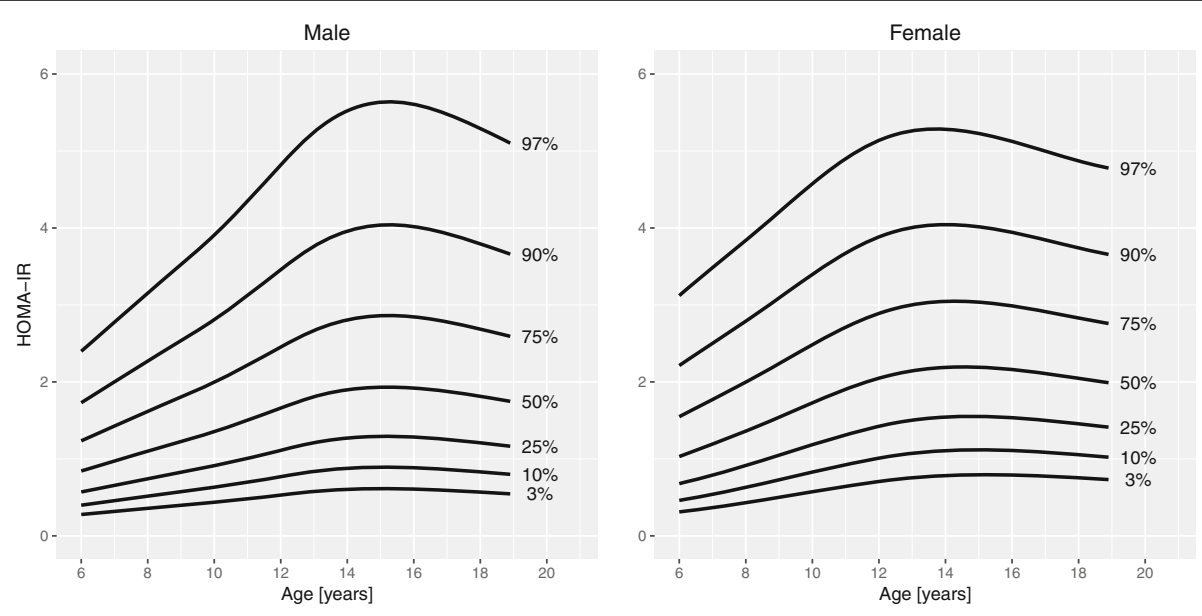

Fig. 5 Percentile curves for homeostasis model assessment insulin resistance (HOMA-IR) levels for male and female Canadian children and youth aged 6 to 19 years
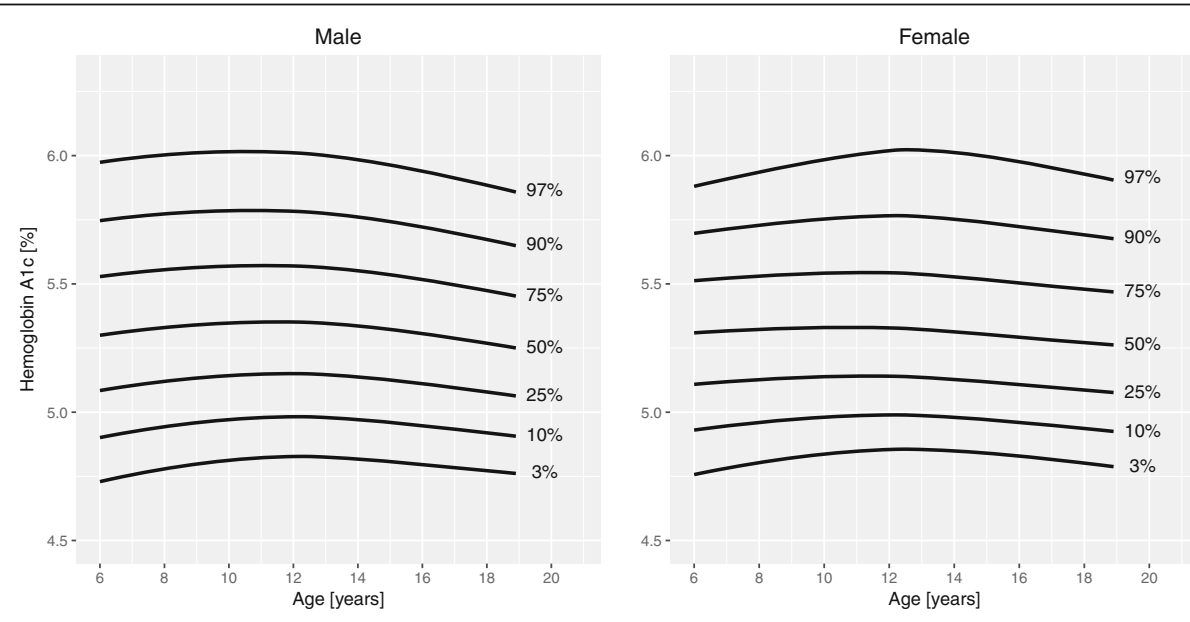

Fig. 6 Percentile curves for hemoglobin A1c levels for male and female Canadian children and youth aged 6 to 19 years 
detail elsewhere [21]. Briefly, the method uses a Box-Cox transformation to normalize the data and models the mean $(\mathrm{M})$, variance $(\mathrm{S})$, and skewness (L) as parameters over age using cubic splines. Centiles and $\mathrm{z}$-scores for the truncated standard normal distribution can then be determined from the three parameters at each age [20]. We calculated the 3rd, 10th, 25th, 50th, 75th, 90th, and 97th centile for each marker. Models were fit to data from respondents up to age 30 years to avoid unusual behaviour of the spline functions near the end of the age range. The goodness of fit for each model was assessed using residual quantile plots ("worm plots") [22]. All calculations were performed using sample weights provided by Statistics Canada to account for the design effect and reduce non-response bias. The statistical software package $\mathrm{R}$ [23] with the gamlss package [24] was used to perform the statistical analyses.

\section{Results}

Sociodemographic characteristics of the sample are summarized in Table 1. Figures 1, 2, 3, 4, 5 and 6 and Tables 2, 3, 4, 5, 6 and 7 show the percentile curves and their values for total cholesterol, HDL cholesterol, triglycerides, insulin, HOMA-IR, and HbA1c.

Total cholesterol curves had a bimodal distribution for both boys and girls. Overall, cholesterol levels were slightly higher in girls than in boys. Median levels at 6 years were $4.1 \mathrm{mmol} / \mathrm{L}$ in girls and $4.0 \mathrm{mmol} / \mathrm{L}$ in boys. In boys, the 50th centile peaked at age 10 years $(4.2 \mathrm{mmol} / \mathrm{L})$. The lowest median cholesterol level in

Table $2 \mathrm{~L}, \mathrm{M}$, and $\mathrm{S}$ values, and percentiles of total cholesterol [mmol/L] by age and sex for Canadian children and youth aged 6 to 19 years

\begin{tabular}{|c|c|c|c|c|c|c|c|c|c|c|c|c|}
\hline Sex & Age & $\mathrm{n}$ & L & $M$ & $S$ & $3 \mathrm{rd}$ & 10th & 25th & 50th & 75th & 90th & 97th \\
\hline \multirow[t]{14}{*}{ Female } & 6 & 203 & 1.2662 & 4.0824 & 0.1676 & 2.73 & 3.18 & 3.61 & 4.08 & 4.54 & 4.94 & 5.32 \\
\hline & 7 & 184 & 1.0339 & 4.1657 & 0.1666 & 2.85 & 3.27 & 3.70 & 4.17 & 4.63 & 5.05 & 5.46 \\
\hline & 8 & 215 & 0.7986 & 4.2255 & 0.1654 & 2.96 & 3.35 & 3.76 & 4.23 & 4.70 & 5.14 & 5.58 \\
\hline & 9 & 233 & 0.5790 & 4.2369 & 0.1645 & 3.01 & 3.38 & 3.78 & 4.24 & 4.72 & 5.17 & 5.63 \\
\hline & 10 & 256 & 0.3985 & 4.2026 & 0.1642 & 3.02 & 3.37 & 3.75 & 4.20 & 4.68 & 5.14 & 5.62 \\
\hline & 11 & 283 & 0.2725 & 4.1427 & 0.1646 & 3.00 & 3.33 & 3.70 & 4.14 & 4.62 & 5.09 & 5.58 \\
\hline & 12 & 187 & 0.2000 & 4.0742 & 0.1658 & 2.95 & 3.28 & 3.64 & 4.07 & 4.55 & 5.02 & 5.51 \\
\hline & 13 & 178 & 0.1564 & 4.0321 & 0.1673 & 2.92 & 3.24 & 3.60 & 4.03 & 4.51 & 4.98 & 5.48 \\
\hline & 14 & 170 & 0.1308 & 4.0222 & 0.1691 & 2.91 & 3.23 & 3.59 & 4.02 & 4.50 & 4.98 & 5.49 \\
\hline & 15 & 175 & 0.1173 & 4.0342 & 0.1717 & 2.90 & 3.23 & 3.59 & 4.03 & 4.53 & 5.01 & 5.54 \\
\hline & 16 & 178 & 0.1121 & 4.0701 & 0.1746 & 2.91 & 3.24 & 3.61 & 4.07 & 4.58 & 5.08 & 5.62 \\
\hline & 17 & 186 & 0.1066 & 4.1326 & 0.1769 & 2.94 & 3.28 & 3.66 & 4.13 & 4.65 & 5.17 & 5.73 \\
\hline & 18 & 168 & 0.1017 & 4.2050 & 0.1788 & 2.99 & 3.33 & 3.72 & 4.21 & 4.74 & 5.27 & 5.85 \\
\hline & 19 & 152 & 0.0901 & 4.2612 & 0.1809 & 3.02 & 3.37 & 3.77 & 4.26 & 4.81 & 5.36 & 5.96 \\
\hline \multirow[t]{14}{*}{ Male } & 6 & 203 & 1.1968 & 4.0369 & 0.1508 & 2.86 & 3.24 & 3.62 & 4.04 & 4.44 & 4.80 & 5.15 \\
\hline & 7 & 211 & 0.8365 & 4.0884 & 0.1523 & 2.95 & 3.30 & 3.67 & 4.09 & 4.51 & 4.90 & 5.29 \\
\hline & 8 & 232 & 0.5410 & 4.1350 & 0.1551 & 3.01 & 3.35 & 3.71 & 4.13 & 4.58 & 4.99 & 5.42 \\
\hline & 9 & 239 & 0.3792 & 4.1691 & 0.1597 & 3.03 & 3.37 & 3.73 & 4.17 & 4.63 & 5.08 & 5.54 \\
\hline & 10 & 263 & 0.3624 & 4.1766 & 0.1651 & 3.00 & 3.35 & 3.73 & 4.18 & 4.66 & 5.12 & 5.61 \\
\hline & 11 & 277 & 0.3556 & 4.1469 & 0.1703 & 2.95 & 3.30 & 3.69 & 4.15 & 4.64 & 5.12 & 5.62 \\
\hline & 12 & 207 & 0.3332 & 4.0711 & 0.1757 & 2.87 & 3.22 & 3.61 & 4.07 & 4.57 & 5.06 & 5.57 \\
\hline & 13 & 207 & 0.3217 & 3.9598 & 0.1801 & 2.77 & 3.12 & 3.50 & 3.96 & 4.46 & 4.95 & 5.46 \\
\hline & 14 & 205 & 0.2648 & 3.8546 & 0.1833 & 2.69 & 3.02 & 3.40 & 3.85 & 4.35 & 4.84 & 5.36 \\
\hline & 15 & 184 & 0.1878 & 3.7910 & 0.1863 & 2.64 & 2.97 & 3.34 & 3.79 & 4.29 & 4.79 & 5.32 \\
\hline & 16 & 208 & 0.1426 & 3.7735 & 0.1886 & 2.62 & 2.95 & 3.32 & 3.77 & 4.28 & 4.79 & 5.33 \\
\hline & 17 & 183 & 0.1015 & 3.7992 & 0.1883 & 2.65 & 2.98 & 3.34 & 3.80 & 4.31 & 4.82 & 5.38 \\
\hline & 18 & 144 & 0.0557 & 3.8565 & 0.1873 & 2.70 & 3.03 & 3.40 & 3.86 & 4.37 & 4.90 & 5.47 \\
\hline & 19 & 139 & 0.0083 & 3.9299 & 0.1869 & 2.76 & 3.09 & 3.46 & 3.93 & 4.46 & 4.99 & 5.58 \\
\hline
\end{tabular}


Table $3 \mathrm{~L}, \mathrm{M}$, and S values, and percentiles of HDL cholesterol [mmol/L] by age and sex for Canadian children and youth aged 6 to 19 years

\begin{tabular}{|c|c|c|c|c|c|c|c|c|c|c|c|c|}
\hline Sex & Age & $n$ & $\mathrm{~L}$ & M & $S$ & $3 r d$ & 10th & 25th & 50th & 75th & 90th & 97th \\
\hline \multirow[t]{14}{*}{ Female } & 6 & 203 & 0.6653 & 1.2995 & 0.2301 & 0.78 & 0.94 & 1.10 & 1.30 & 1.51 & 1.70 & 1.90 \\
\hline & 7 & 184 & 0.5810 & 1.3513 & 0.2315 & 0.82 & 0.98 & 1.15 & 1.35 & 1.57 & 1.78 & 1.99 \\
\hline & 8 & 215 & 0.4894 & 1.3925 & 0.2317 & 0.85 & 1.01 & 1.18 & 1.39 & 1.62 & 1.84 & 2.07 \\
\hline & 9 & 233 & 0.4025 & 1.4128 & 0.2304 & 0.88 & 1.03 & 1.20 & 1.41 & 1.64 & 1.87 & 2.11 \\
\hline & 10 & 256 & 0.3317 & 1.4069 & 0.2269 & 0.89 & 1.04 & 1.20 & 1.41 & 1.63 & 1.86 & 2.10 \\
\hline & 11 & 283 & 0.2946 & 1.3835 & 0.2222 & 0.89 & 1.03 & 1.19 & 1.38 & 1.60 & 1.82 & 2.05 \\
\hline & 12 & 187 & 0.2932 & 1.3527 & 0.2180 & 0.87 & 1.01 & 1.16 & 1.35 & 1.56 & 1.77 & 1.99 \\
\hline & 13 & 178 & 0.2666 & 1.3372 & 0.2152 & 0.87 & 1.00 & 1.15 & 1.34 & 1.54 & 1.74 & 1.96 \\
\hline & 14 & 170 & 0.1991 & 1.3404 & 0.2145 & 0.88 & 1.01 & 1.16 & 1.34 & 1.55 & 1.75 & 1.98 \\
\hline & 15 & 175 & 0.1202 & 1.3502 & 0.2156 & 0.89 & 1.02 & 1.17 & 1.35 & 1.56 & 1.77 & 2.01 \\
\hline & 16 & 178 & 0.0477 & 1.3590 & 0.2180 & 0.90 & 1.03 & 1.17 & 1.36 & 1.57 & 1.79 & 2.04 \\
\hline & 17 & 186 & -0.0261 & 1.3764 & 0.2213 & 0.91 & 1.04 & 1.19 & 1.38 & 1.60 & 1.83 & 2.09 \\
\hline & 18 & 168 & -0.0758 & 1.4019 & 0.2258 & 0.92 & 1.05 & 1.20 & 1.40 & 1.63 & 1.88 & 2.16 \\
\hline & 19 & 152 & -0.0551 & 1.4209 & 0.2316 & 0.92 & 1.06 & 1.22 & 1.42 & 1.66 & 1.92 & 2.21 \\
\hline \multirow[t]{14}{*}{ Male } & 6 & 203 & 0.8228 & 1.4346 & 0.2226 & 0.86 & 1.04 & 1.22 & 1.43 & 1.65 & 1.85 & 2.06 \\
\hline & 7 & 211 & 0.6385 & 1.4440 & 0.2207 & 0.89 & 1.06 & 1.23 & 1.44 & 1.66 & 1.87 & 2.09 \\
\hline & 8 & 232 & 0.4626 & 1.4509 & 0.2215 & 0.91 & 1.07 & 1.24 & 1.45 & 1.68 & 1.89 & 2.12 \\
\hline & 9 & 239 & 0.3069 & 1.4533 & 0.2256 & 0.92 & 1.07 & 1.24 & 1.45 & 1.69 & 1.92 & 2.17 \\
\hline & 10 & 263 & 0.1937 & 1.4465 & 0.2296 & 0.92 & 1.07 & 1.24 & 1.45 & 1.68 & 1.93 & 2.19 \\
\hline & 11 & 277 & 0.1465 & 1.4230 & 0.2297 & 0.91 & 1.05 & 1.22 & 1.42 & 1.66 & 1.90 & 2.16 \\
\hline & 12 & 207 & 0.1687 & 1.3827 & 0.2281 & 0.89 & 1.02 & 1.18 & 1.38 & 1.61 & 1.84 & 2.09 \\
\hline & 13 & 207 & 0.2352 & 1.3335 & 0.2266 & 0.85 & 0.99 & 1.14 & 1.33 & 1.55 & 1.77 & 2.00 \\
\hline & 14 & 205 & 0.3157 & 1.2882 & 0.2251 & 0.82 & 0.95 & 1.10 & 1.29 & 1.49 & 1.70 & 1.92 \\
\hline & 15 & 184 & 0.3915 & 1.2523 & 0.2244 & 0.79 & 0.92 & 1.07 & 1.25 & 1.45 & 1.64 & 1.85 \\
\hline & 16 & 208 & 0.4549 & 1.2270 & 0.2256 & 0.77 & 0.90 & 1.05 & 1.23 & 1.42 & 1.61 & 1.81 \\
\hline & 17 & 183 & 0.4843 & 1.2122 & 0.2260 & 0.75 & 0.89 & 1.03 & 1.21 & 1.40 & 1.59 & 1.78 \\
\hline & 18 & 144 & 0.4698 & 1.2062 & 0.2245 & 0.75 & 0.89 & 1.03 & 1.21 & 1.40 & 1.58 & 1.77 \\
\hline & 19 & 139 & 0.4299 & 1.2084 & 0.2234 & 0.76 & 0.89 & 1.03 & 1.21 & 1.40 & 1.58 & 1.78 \\
\hline
\end{tabular}

Abbreviations: $L$ lambda (skewness); $M$ mu (mean); $S$ sigma (variance)

boys was seen at 16 years of age $(3.8 \mathrm{mmol} / \mathrm{L})$, after which it increased to $3.9 \mathrm{mmol} / \mathrm{L}$ at 19 years. In girls, the 50th centile for cholesterol had a peak at 9 years $(4.2 \mathrm{mmol} / \mathrm{L})$, decreased to a trough at 15 years $(4.0 \mathrm{mmol} / \mathrm{L})$ and increased again to $4.3 \mathrm{mmol} / \mathrm{L}$ at 19 years of age. HDL cholesterol showed a bimodal distribution in girls, but only one peak in boys. In boys, the median levels were highest before 11 years $(1.5 \mathrm{mmol} / \mathrm{L})$ and then steadily declined until 19 years $(1.2 \mathrm{mmol} / \mathrm{L})$. Median HDL cholesterol in girls peaked at age 10 years $(1.4 \mathrm{mmol} / \mathrm{L})$ and after a trough $(1.3 \mathrm{mmol} / \mathrm{L})$ increased again to $1.4 \mathrm{mmol} / \mathrm{L}$ at 19 years. Median levels of triglycerides exhibited a steady linear increase from 6 years $(0.7 \mathrm{mmol} / \mathrm{L})$ to 19 years $(0.9 \mathrm{mmol} / \mathrm{L})$ for both sexes. Insulin levels were overall higher in girls than in boys. For both sexes, median levels increased until about 14 years of age (62 and $72 \mathrm{pmol} / \mathrm{L}$ in boys and girls, respectively), after which they slightly decreased to $57 \mathrm{pmol} / \mathrm{L}$ in boys and $70 \mathrm{pmol} / \mathrm{L}$ in girls at 19 years of age. Centile curves for HOMA-IR largely mirrored those for insulin with the 50th percentile peaking at 15 years for both sexes (1.9 for boys and 2.2 for girls). Median HbA1c levels held nearly constant around 5.3\% from 6 to 19 years for both sexes.

\section{Discussion}

The objective of this study was to develop percentile curves for total cholesterol, HDL cholesterol, triglycerides, insulin, HOMA-IR, and HbA1c in a population-based 
Table $4 \mathrm{~L}, \mathrm{M}$, and S values, and percentiles of triglycerides [mmol/L] by age and sex for Canadian children and youth aged 6 to 19 years

\begin{tabular}{|c|c|c|c|c|c|c|c|c|c|c|c|c|}
\hline Sex & Age & $\mathrm{n}$ & $\mathrm{L}$ & M & $\mathrm{S}$ & $3 r d$ & 10th & 25th & 50th & 75th & 90th & 97th \\
\hline \multirow[t]{14}{*}{ Female } & 6 & 78 & -0.0198 & 0.7390 & 0.3934 & 0.35 & 0.45 & 0.57 & 0.74 & 0.96 & 1.23 & 1.56 \\
\hline & 7 & 85 & -0.0360 & 0.7567 & 0.3932 & 0.36 & 0.46 & 0.58 & 0.76 & 0.99 & 1.26 & 1.60 \\
\hline & 8 & 98 & -0.0522 & 0.7744 & 0.3931 & 0.37 & 0.47 & 0.60 & 0.77 & 1.01 & 1.29 & 1.65 \\
\hline & 9 & 127 & -0.0680 & 0.7922 & 0.3931 & 0.39 & 0.48 & 0.61 & 0.79 & 1.04 & 1.32 & 1.69 \\
\hline & 10 & 114 & -0.0821 & 0.8100 & 0.3931 & 0.40 & 0.49 & 0.62 & 0.81 & 1.06 & 1.35 & 1.74 \\
\hline & 11 & 139 & -0.0928 & 0.8273 & 0.3932 & 0.40 & 0.51 & 0.64 & 0.83 & 1.08 & 1.39 & 1.78 \\
\hline & 12 & 93 & -0.0987 & 0.8438 & 0.3935 & 0.41 & 0.52 & 0.65 & 0.84 & 1.10 & 1.42 & 1.82 \\
\hline & 13 & 88 & -0.0996 & 0.8593 & 0.3942 & 0.42 & 0.52 & 0.66 & 0.86 & 1.13 & 1.44 & 1.86 \\
\hline & 14 & 88 & -0.0958 & 0.8738 & 0.3954 & 0.43 & 0.53 & 0.67 & 0.87 & 1.14 & 1.47 & 1.89 \\
\hline & 15 & 91 & -0.0874 & 0.8874 & 0.3972 & 0.43 & 0.54 & 0.68 & 0.89 & 1.16 & 1.49 & 1.92 \\
\hline & 16 & 91 & -0.0746 & 0.9003 & 0.3996 & 0.43 & 0.54 & 0.69 & 0.90 & 1.18 & 1.52 & 1.95 \\
\hline & 17 & 89 & -0.0581 & 0.9125 & 0.4026 & 0.43 & 0.55 & 0.70 & 0.91 & 1.20 & 1.54 & 1.98 \\
\hline & 18 & 74 & -0.0389 & 0.9240 & 0.4061 & 0.44 & 0.55 & 0.70 & 0.92 & 1.22 & 1.56 & 2.01 \\
\hline & 19 & 72 & -0.0180 & 0.9346 & 0.4102 & 0.43 & 0.55 & 0.71 & 0.93 & 1.23 & 1.58 & 2.03 \\
\hline \multirow[t]{14}{*}{ Male } & 6 & 102 & -0.2816 & 0.6737 & 0.4053 & 0.34 & 0.41 & 0.52 & 0.67 & 0.90 & 1.18 & 1.59 \\
\hline & 7 & 98 & -0.2348 & 0.6849 & 0.4111 & 0.34 & 0.42 & 0.52 & 0.68 & 0.91 & 1.20 & 1.61 \\
\hline & 8 & 116 & -0.1881 & 0.6961 & 0.4169 & 0.33 & 0.42 & 0.53 & 0.70 & 0.93 & 1.22 & 1.63 \\
\hline & 9 & 120 & -0.1418 & 0.7075 & 0.4227 & 0.33 & 0.42 & 0.54 & 0.71 & 0.95 & 1.24 & 1.64 \\
\hline & 10 & 133 & -0.0967 & 0.7195 & 0.4281 & 0.33 & 0.42 & 0.54 & 0.72 & 0.96 & 1.26 & 1.66 \\
\hline & 11 & 135 & -0.0544 & 0.7322 & 0.4329 & 0.33 & 0.42 & 0.55 & 0.73 & 0.98 & 1.29 & 1.68 \\
\hline & 12 & 96 & -0.0165 & 0.7459 & 0.4368 & 0.33 & 0.43 & 0.56 & 0.75 & 1.00 & 1.31 & 1.71 \\
\hline & 13 & 112 & 0.0157 & 0.7606 & 0.4395 & 0.33 & 0.43 & 0.57 & 0.76 & 1.02 & 1.33 & 1.73 \\
\hline & 14 & 97 & 0.0421 & 0.7763 & 0.4412 & 0.33 & 0.44 & 0.58 & 0.78 & 1.04 & 1.36 & 1.75 \\
\hline & 15 & 93 & 0.0630 & 0.7927 & 0.4422 & 0.34 & 0.45 & 0.59 & 0.79 & 1.07 & 1.38 & 1.78 \\
\hline & 16 & 103 & 0.0788 & 0.8098 & 0.4428 & 0.34 & 0.45 & 0.60 & 0.81 & 1.09 & 1.41 & 1.81 \\
\hline & 17 & 92 & 0.0899 & 0.8275 & 0.4431 & 0.35 & 0.46 & 0.61 & 0.83 & 1.11 & 1.44 & 1.85 \\
\hline & 18 & 78 & 0.0968 & 0.8457 & 0.4435 & 0.35 & 0.47 & 0.62 & 0.85 & 1.14 & 1.47 & 1.89 \\
\hline & 19 & 74 & 0.0999 & 0.8642 & 0.4441 & 0.36 & 0.48 & 0.64 & 0.86 & 1.16 & 1.50 & 1.93 \\
\hline
\end{tabular}

Abbreviations: $L$ lambda (skewness); $M$ mu (mean); $S$ sigma (variance)

sample of Canadian children and youth. We found ageand sex-related differences in blood levels for all markers except for HbA1c.

A bimodal shape of the centile curves for total cholesterol levels has been described in various Western populations $[9,11,13,25]$. A pre-adolescent peak at around 8 to 10 years of age that is more pronounced in boys is followed by a decrease during adolescence and another peak in late adolescence and young adulthood. The same pattern, but without a post-pubertal rise in boys, can be seen for HDL cholesterol $[9,11,13]$. The pubertal trough of cholesterol levels may be the result of the well described insulin resistance during puberty [26]. Clinicians should be aware of these physiologic changes when interpreting cholesterol levels. However, it should also be acknowledged that median levels of cholesterol in our study as well as in other studies varied by $10 \%$ or less in either direction during childhood and adolescence $[9,11,13,25]$.

Median triglyceride levels in our sample showed a nearly linear increase by about $30 \%$ from around 0.7 to $0.9 \mathrm{mmol} / \mathrm{L}$ in both sexes during childhood and adolescence. Some investigators previously described a bimodal pattern in girls with peaks at around 12 and 19 years of age $[9,11]$, while others also reported the linear increase we found [13]. These differences may be explained by different degrees of smoothing applied during the modeling process.

Median fasting insulin levels were higher in girls than in boys, and levels in both sexes peaked at around 15 years of age followed by a slight decrease 
Table 5 L, M, and S values, and percentiles of insulin [pmol/L] by age and sex for Canadian children and youth aged 6 to 19 years

\begin{tabular}{|c|c|c|c|c|c|c|c|c|c|c|c|c|}
\hline Sex & Age & $n$ & $\mathrm{~L}$ & $M$ & $S$ & $3 r d$ & 10th & 25th & 50th & 75th & 90th & 97th \\
\hline \multirow[t]{14}{*}{ Female } & 6 & 74 & 0.0810 & 36.8032 & 0.5822 & 11.69 & 17.05 & 24.69 & 36.80 & 54.17 & 75.94 & 105.08 \\
\hline & 7 & 85 & 0.0832 & 41.9738 & 0.5661 & 13.77 & 19.86 & 28.47 & 41.97 & 61.13 & 84.90 & 116.43 \\
\hline & 8 & 95 & 0.0861 & 47.1805 & 0.5505 & 15.95 & 22.79 & 32.35 & 47.18 & 68.00 & 93.58 & 127.19 \\
\hline & 9 & 124 & 0.0909 & 52.4480 & 0.5352 & 18.25 & 25.83 & 36.33 & 52.45 & 74.82 & 102.02 & 137.42 \\
\hline & 10 & 110 & 0.0997 & 57.6269 & 0.5201 & 20.59 & 28.91 & 40.32 & 57.63 & 81.35 & 109.87 & 146.55 \\
\hline & 11 & 136 & 0.1121 & 62.4301 & 0.5044 & 22.90 & 31.91 & 44.13 & 62.43 & 87.17 & 116.52 & 153.78 \\
\hline & 12 & 92 & 0.1263 & 66.5218 & 0.4884 & 25.06 & 34.66 & 47.52 & 66.52 & 91.86 & 121.50 & 158.64 \\
\hline & 13 & 88 & 0.1414 & 69.6293 & 0.4734 & 26.89 & 36.93 & 50.22 & 69.63 & 95.15 & 124.61 & 161.05 \\
\hline & 14 & 86 & 0.1553 & 71.6632 & 0.4607 & 28.26 & 38.58 & 52.12 & 71.66 & 97.07 & 126.08 & 161.58 \\
\hline & 15 & 86 & 0.1652 & 72.6769 & 0.4515 & 29.11 & 39.56 & 53.17 & 72.68 & 97.82 & 126.30 & 160.90 \\
\hline & 16 & 90 & 0.1703 & 72.8110 & 0.4465 & 29.42 & 39.88 & 53.45 & 72.81 & 97.67 & 125.70 & 159.62 \\
\hline & 17 & 86 & 0.1707 & 72.2282 & 0.4460 & 29.21 & 39.58 & 53.04 & 72.23 & 96.85 & 124.61 & 158.18 \\
\hline & 18 & 74 & 0.1665 & 71.1378 & 0.4500 & 28.57 & 38.80 & 52.10 & 71.14 & 95.65 & 123.38 & 157.03 \\
\hline & 19 & 71 & 0.1592 & 69.7465 & 0.4583 & 27.60 & 37.65 & 50.80 & 69.75 & 94.31 & 122.28 & 156.42 \\
\hline \multirow[t]{14}{*}{ Male } & 6 & 98 & -0.0050 & 29.2964 & 0.5269 & 10.90 & 14.93 & 20.54 & 29.30 & 41.81 & 57.62 & 79.12 \\
\hline & 7 & 95 & -0.0012 & 33.2034 & 0.5309 & 12.24 & 16.82 & 23.21 & 33.20 & 47.50 & 65.58 & 90.17 \\
\hline & 8 & 116 & 0.0028 & 37.0587 & 0.5348 & 13.54 & 18.66 & 25.83 & 37.06 & 53.14 & 73.49 & 101.18 \\
\hline & 9 & 118 & 0.0065 & 40.8164 & 0.5385 & 14.78 & 20.44 & 28.37 & 40.82 & 58.67 & 81.26 & 112.00 \\
\hline & 10 & 130 & 0.0097 & 44.7223 & 0.5419 & 16.06 & 22.28 & 31.01 & 44.72 & 64.42 & 89.36 & 123.31 \\
\hline & 11 & 129 & 0.0126 & 49.2160 & 0.5451 & 17.54 & 24.40 & 34.05 & 49.22 & 71.02 & 98.66 & 136.29 \\
\hline & 12 & 95 & 0.0153 & 54.1827 & 0.5477 & 19.18 & 26.75 & 37.41 & 54.18 & 78.32 & 108.91 & 150.58 \\
\hline & 13 & 110 & 0.0178 & 58.6671 & 0.5499 & 20.65 & 28.87 & 40.44 & 58.67 & 84.91 & 118.19 & 163.49 \\
\hline & 14 & 95 & 0.0203 & 61.7110 & 0.5519 & 21.61 & 30.27 & 42.47 & 61.71 & 89.42 & 124.55 & 172.38 \\
\hline & 15 & 91 & 0.0232 & 62.9131 & 0.5539 & 21.91 & 30.75 & 43.23 & 62.91 & 91.27 & 127.21 & 176.13 \\
\hline & 16 & 102 & 0.0271 & 62.5506 & 0.5561 & 21.65 & 30.46 & 42.90 & 62.55 & 90.85 & 126.71 & 175.45 \\
\hline & 17 & 91 & 0.0318 & 61.1922 & 0.5583 & 21.03 & 29.67 & 41.90 & 61.19 & 88.97 & 124.15 & 171.90 \\
\hline & 18 & 76 & 0.0370 & 59.3041 & 0.5602 & 20.25 & 28.65 & 40.54 & 59.30 & 86.30 & 120.44 & 166.70 \\
\hline & 19 & 74 & 0.0431 & 57.1325 & 0.5615 & 19.39 & 27.51 & 39.00 & 57.13 & 83.18 & 116.05 & 160.46 \\
\hline
\end{tabular}

Abbreviations: $L$ lambda (skewness); $M$ mu (mean); $S$ sigma (variance)

towards late adolescence. Similar age and sex differences have also been reported by others [13, 27]. The peak in puberty reflects the physiologically reduced insulin sensitivity and concomitant increase in insulin secretion during that period $[28,29]$. The median insulin levels in our study and others $[12,13,27]$ varied with pubertal levels ranging from 52 to $63 \mathrm{pmol} / \mathrm{L}$ in boys and from 65 to $73 \mathrm{pmol} / \mathrm{L}$ in girls. These differences may be explained by differences in the insulin assay used [30] or differences in the body composition, ethnicity, and puberty stage of the children in the sample. The shape of the HOMA-IR curves was similar to those for fasting insulin. Schwartz et al. found a significant correlation between fasting insulin and HOMA-IR but found both only modestly correlated with the insulin resistance measurement gold standard, the euglycemic-hyperinsulinemic glucose clamp [31]. HOMA-IR still is among the most commonly used surrogate measure of insulin resistance to date. Given the variation over sex and age, in particular the physiologic insulin resistance in puberty, the use of an age- and sex-specific percentile-based cutoff for HOMA-IR is warranted. Unfortunately, such a cutoff has not been established to date [32].

Glycosylated hemoglobin or $\mathrm{HbA1c}$ is an established marker for long-term glycemic control in patients with diabetes [33]. HbA1c has been proposed as a screening tool for undiagnosed diabetes in adults [6] and children with overweight or obesity [8], but the evidence is still very limited. We found very little change in HbA1c levels from childhood to late adolescence, and there was no difference between the sexes. 
Table 6 L, M, and S values, and percentiles of HOMA-IR by age and sex for Canadian children and youth aged 6 to 19 years

\begin{tabular}{|c|c|c|c|c|c|c|c|c|c|c|c|c|}
\hline Sex & Age & $\mathrm{n}$ & $\mathrm{L}$ & $M$ & $S$ & $3 \mathrm{rd}$ & 10th & 25th & 50th & 75th & 90th & 97th \\
\hline \multirow[t]{14}{*}{ Female } & 6 & 74 & 0.0680 & 1.0304 & 0.6120 & 0.31 & 0.46 & 0.68 & 1.03 & 1.55 & 2.21 & 3.12 \\
\hline & 7 & 85 & 0.0816 & 1.1930 & 0.5955 & 0.37 & 0.54 & 0.79 & 1.19 & 1.77 & 2.50 & 3.48 \\
\hline & 8 & 95 & 0.0953 & 1.3607 & 0.5795 & 0.43 & 0.63 & 0.91 & 1.36 & 2.00 & 2.79 & 3.84 \\
\hline & 9 & 124 & 0.1090 & 1.5413 & 0.5642 & 0.50 & 0.73 & 1.04 & 1.54 & 2.24 & 3.09 & 4.21 \\
\hline & 10 & 110 & 0.1226 & 1.7284 & 0.5496 & 0.57 & 0.83 & 1.18 & 1.73 & 2.48 & 3.40 & 4.57 \\
\hline & 11 & 136 & 0.1354 & 1.9044 & 0.5360 & 0.64 & 0.93 & 1.31 & 1.90 & 2.71 & 3.67 & 4.90 \\
\hline & 12 & 92 & 0.1467 & 2.0481 & 0.5235 & 0.71 & 1.01 & 1.43 & 2.05 & 2.89 & 3.88 & 5.14 \\
\hline & 13 & 88 & 0.1557 & 2.1435 & 0.5125 & 0.75 & 1.07 & 1.50 & 2.14 & 3.00 & 4.01 & 5.26 \\
\hline & 14 & 86 & 0.1619 & 2.1886 & 0.5036 & 0.78 & 1.11 & 1.54 & 2.19 & 3.05 & 4.04 & 5.28 \\
\hline & 15 & 86 & 0.1645 & 2.1908 & 0.4970 & 0.79 & 1.12 & 1.55 & 2.19 & 3.04 & 4.02 & 5.23 \\
\hline & 16 & 90 & 0.1635 & 2.1612 & 0.4930 & 0.79 & 1.11 & 1.54 & 2.16 & 2.99 & 3.94 & 5.12 \\
\hline & 17 & 86 & 0.1591 & 2.1092 & 0.4916 & 0.78 & 1.09 & 1.50 & 2.11 & 2.91 & 3.84 & 5.00 \\
\hline & 18 & 74 & 0.1514 & 2.0465 & 0.4928 & 0.75 & 1.05 & 1.46 & 2.05 & 2.83 & 3.74 & 4.87 \\
\hline & 19 & 71 & 0.1411 & 1.9837 & 0.4965 & 0.73 & 1.02 & 1.41 & 1.98 & 2.75 & 3.65 & 4.77 \\
\hline \multirow[t]{14}{*}{ Male } & 6 & 98 & 0.0558 & 0.8427 & 0.5725 & 0.28 & 0.40 & 0.57 & 0.84 & 1.23 & 1.73 & 2.40 \\
\hline & 7 & 95 & 0.0576 & 0.9718 & 0.5752 & 0.32 & 0.46 & 0.66 & 0.97 & 1.43 & 2.00 & 2.78 \\
\hline & 8 & 116 & 0.0595 & 1.1004 & 0.5778 & 0.36 & 0.52 & 0.74 & 1.10 & 1.62 & 2.27 & 3.15 \\
\hline & 9 & 118 & 0.0610 & 1.2255 & 0.5802 & 0.40 & 0.57 & 0.82 & 1.23 & 1.80 & 2.54 & 3.52 \\
\hline & 10 & 130 & 0.0617 & 1.3545 & 0.5823 & 0.44 & 0.63 & 0.91 & 1.35 & 2.00 & 2.81 & 3.91 \\
\hline & 11 & 129 & 0.0619 & 1.5014 & 0.5841 & 0.48 & 0.70 & 1.01 & 1.50 & 2.22 & 3.12 & 4.35 \\
\hline & 12 & 95 & 0.0618 & 1.6614 & 0.5855 & 0.53 & 0.77 & 1.11 & 1.66 & 2.45 & 3.46 & 4.82 \\
\hline & 13 & 110 & 0.0618 & 1.8031 & 0.5864 & 0.58 & 0.84 & 1.21 & 1.80 & 2.67 & 3.76 & 5.24 \\
\hline & 14 & 95 & 0.0619 & 1.8974 & 0.5873 & 0.60 & 0.88 & 1.27 & 1.90 & 2.81 & 3.96 & 5.52 \\
\hline & 15 & 91 & 0.0627 & 1.9332 & 0.5883 & 0.61 & 0.89 & 1.29 & 1.93 & 2.86 & 4.04 & 5.63 \\
\hline & 16 & 102 & 0.0646 & 1.9204 & 0.5896 & 0.61 & 0.89 & 1.28 & 1.92 & 2.84 & 4.02 & 5.60 \\
\hline & 17 & 91 & 0.0673 & 1.8754 & 0.5910 & 0.59 & 0.86 & 1.25 & 1.88 & 2.78 & 3.93 & 5.48 \\
\hline & 18 & 76 & 0.0705 & 1.8126 & 0.5922 & 0.57 & 0.83 & 1.21 & 1.81 & 2.69 & 3.80 & 5.30 \\
\hline & 19 & 74 & 0.0747 & 1.7406 & 0.5929 & 0.54 & 0.80 & 1.16 & 1.74 & 2.58 & 3.64 & 5.08 \\
\hline
\end{tabular}

Abbreviations: $L$ lambda (skewness); $M$ mu (mean); $S$ sigma (variance)

To the best of our knowledge, only three previous studies have examined HbA1c levels during childhood [12, 14, 34]. Only Peplies et al. in the European IDEFICS cohort developed percentile curves to describe the changes in levels across age and found a $15 \%$ increase in median HbA1c levels between 7 and 11 years for both sexes [12].

The strengths of our study include the use of a large population-based sample and the use of standardized protocols and procedures for the measurement of the cardiometabolic marker levels. A shortcoming of the use of cross-sectional data is that it is not clear if the trajectories of individual children follow this pattern; longitudinal data may be more accurate in describing age-related changes but are considerably more resource intensive to collect at the population level. Due to the relatively small proportion of visible minority children in the sample $(<20 \%)$, we were not able to investigate ethnic differences in marker levels and trajectories. Another limitation of our study is that we were unable to take puberty stage, which may influence insulin and lipid levels, into account as this information was not available in the CHMS. By contrast to some of the previous studies in this area, we did not restrict our analysis to children with a healthy weight $[9,12]$, as our goal was to describe population-based trajectories. Since the inclusion of overweight and obese children in our sample may have influenced lipid and insulin levels, our percentiles cannot be considered as reference values. 
Table $7 \mathrm{~L}, \mathrm{M}$, and S values, and percentiles of hemoglobin A1c by age and sex for Canadian children and youth aged 6 to 19 years

\begin{tabular}{|c|c|c|c|c|c|c|c|c|c|c|c|c|}
\hline Sex & Age & $n$ & $L$ & $M$ & $S$ & $3 \mathrm{rd}$ & 10th & 25th & 50th & 75th & 90th & 97th \\
\hline \multirow[t]{14}{*}{ Female } & 6 & 192 & 0.6293 & 0.0531 & 0.0565 & 0.0476 & 0.0493 & 0.0511 & 0.0531 & 0.0552 & 0.0570 & 0.0589 \\
\hline & 7 & 178 & 0.0341 & 0.0532 & 0.0563 & 0.0478 & 0.0495 & 0.0512 & 0.0532 & 0.0552 & 0.0571 & 0.0591 \\
\hline & 8 & 204 & -0.5398 & 0.0532 & 0.0561 & 0.0480 & 0.0496 & 0.0513 & 0.0532 & 0.0553 & 0.0573 & 0.0593 \\
\hline & 9 & 224 & -1.0745 & 0.0533 & 0.0560 & 0.0482 & 0.0497 & 0.0513 & 0.0533 & 0.0553 & 0.0574 & 0.0596 \\
\hline & 10 & 248 & -1.5959 & 0.0533 & 0.0560 & 0.0484 & 0.0498 & 0.0514 & 0.0533 & 0.0554 & 0.0575 & 0.0598 \\
\hline & 11 & 275 & -2.1144 & 0.0533 & 0.0559 & 0.0485 & 0.0499 & 0.0514 & 0.0533 & 0.0554 & 0.0576 & 0.0600 \\
\hline & 12 & 184 & -2.5527 & 0.0533 & 0.0558 & 0.0486 & 0.0499 & 0.0514 & 0.0533 & 0.0554 & 0.0577 & 0.0602 \\
\hline & 13 & 172 & -2.8025 & 0.0532 & 0.0557 & 0.0486 & 0.0499 & 0.0514 & 0.0532 & 0.0554 & 0.0576 & 0.0603 \\
\hline & 14 & 166 & -2.8388 & 0.0531 & 0.0555 & 0.0485 & 0.0498 & 0.0513 & 0.0531 & 0.0553 & 0.0575 & 0.0601 \\
\hline & 15 & 167 & -2.7545 & 0.0530 & 0.0553 & 0.0484 & 0.0497 & 0.0512 & 0.0530 & 0.0551 & 0.0574 & 0.0599 \\
\hline & 16 & 172 & -2.6052 & 0.0529 & 0.0551 & 0.0483 & 0.0496 & 0.0511 & 0.0529 & 0.0550 & 0.0572 & 0.0597 \\
\hline & 17 & 177 & -2.4044 & 0.0528 & 0.0550 & 0.0481 & 0.0495 & 0.0509 & 0.0528 & 0.0549 & 0.0570 & 0.0595 \\
\hline & 18 & 165 & -2.1906 & 0.0527 & 0.0550 & 0.0480 & 0.0494 & 0.0509 & 0.0527 & 0.0548 & 0.0569 & 0.0593 \\
\hline & 19 & 149 & -2.0342 & 0.0527 & 0.0552 & 0.0479 & 0.0493 & 0.0508 & 0.0527 & 0.0548 & 0.0569 & 0.0592 \\
\hline \multirow[t]{14}{*}{ Male } & 6 & 195 & -0.4642 & 0.0530 & 0.0619 & 0.0473 & 0.0490 & 0.0509 & 0.0530 & 0.0553 & 0.0575 & 0.0598 \\
\hline & 7 & 205 & -0.5917 & 0.0532 & 0.0612 & 0.0476 & 0.0492 & 0.0510 & 0.0532 & 0.0554 & 0.0576 & 0.0599 \\
\hline & 8 & 227 & -0.7384 & 0.0533 & 0.0605 & 0.0478 & 0.0494 & 0.0512 & 0.0533 & 0.0555 & 0.0577 & 0.0600 \\
\hline & 9 & 229 & -0.8769 & 0.0534 & 0.0598 & 0.0480 & 0.0496 & 0.0513 & 0.0534 & 0.0556 & 0.0578 & 0.0601 \\
\hline & 10 & 256 & -0.9903 & 0.0535 & 0.0591 & 0.0481 & 0.0497 & 0.0514 & 0.0535 & 0.0557 & 0.0578 & 0.0601 \\
\hline & 11 & 267 & -1.0740 & 0.0535 & 0.0584 & 0.0482 & 0.0498 & 0.0515 & 0.0535 & 0.0557 & 0.0579 & 0.0602 \\
\hline & 12 & 204 & -1.1009 & 0.0535 & 0.0580 & 0.0483 & 0.0498 & 0.0515 & 0.0535 & 0.0557 & 0.0578 & 0.0601 \\
\hline & 13 & 201 & -1.0887 & 0.0535 & 0.0577 & 0.0483 & 0.0498 & 0.0515 & 0.0535 & 0.0556 & 0.0578 & 0.0600 \\
\hline & 14 & 203 & -1.0467 & 0.0534 & 0.0574 & 0.0482 & 0.0497 & 0.0514 & 0.0534 & 0.0555 & 0.0576 & 0.0599 \\
\hline & 15 & 180 & -1.0081 & 0.0532 & 0.0571 & 0.0481 & 0.0496 & 0.0512 & 0.0532 & 0.0554 & 0.0574 & 0.0596 \\
\hline & 16 & 205 & -0.9894 & 0.0530 & 0.0567 & 0.0479 & 0.0494 & 0.0511 & 0.0530 & 0.0551 & 0.0572 & 0.0594 \\
\hline & 17 & 180 & -1.0085 & 0.0528 & 0.0561 & 0.0478 & 0.0493 & 0.0509 & 0.0528 & 0.0549 & 0.0569 & 0.0591 \\
\hline & 18 & 138 & -1.0586 & 0.0527 & 0.0555 & 0.0477 & 0.0492 & 0.0508 & 0.0527 & 0.0547 & 0.0567 & 0.0588 \\
\hline & 19 & 135 & -1.1174 & 0.0526 & 0.0549 & 0.0477 & 0.0491 & 0.0507 & 0.0526 & 0.0546 & 0.0566 & 0.0587 \\
\hline
\end{tabular}

Abbreviations: $L$ lambda (skewness); $M$ mu (mean); $S$ sigma (variance)

\section{Conclusions}

Our study has developed percentile curves for cardiometabolic disease markers in Canadian children and adolescents. We have demonstrated age- and sex-related differences in marker levels for lipids, insulin, and HOMA-IR that should be considered when evaluating these markers in children and adolescents.

\section{Abbreviations}

CHMS: Canadian Health Measures Survey; CVD: Cardiovascular disease; HbA1c: Hemoglobin A1c; HDL: High-density lipoprotein; HOMA-IR: Homeostasis model assessment insulin resistance; LMS: Lambda Mu Sigma

\section{Acknowledgements}

The analysis presented in this paper was conducted at the Atlantic Research Data Centre, which is part of the Canadian Research Data Centre Network (CRDCN). The services and activities provided by the Atlantic Research Data Centre are made possible by the financial or in-kind support of the SSHRC, the CIHR, the CFI, Statistics Canada, and Dalhousie University. The views expressed in this paper do not necessarily represent the views of the CRDCN or its partners.

\section{Funding}

This work was supported by an IWK Health Centre (http://www.iwk.nshealth.ca) Establishment Grant awarded to Dr. Stefan Kuhle (FRN 09020). The funder had no role in the design of the study and collection, analysis, and interpretation of data and in writing the manuscript.

\section{Availability of data and materials}

The data that support the findings of this study are available from Statistics Canada through the Statistics Canada Research Data Centres program to researchers who meet the criteria for access to confidential data. The application process is described at http://www.statcan.gc.ca/eng/rdc/process In brief, researchers submit an application form and project proposal to the Statistics Canada Research Data Centres Program. Upon approval they have to undergo a security check. Once completed, they get access to one of the Research Data Centres in Canada to analyze the data. Only aggregated data can be released, and all output produced at the centres must be vetted by a Statistics Canada analyst before release.

\section{Authors' contributions}

NA, BM, DCH, and SK conceived and designed the experiments. BM and SK analyzed the data. NA wrote the manuscript. BM, DCH, and SK critically revised the manuscript. All authors read and approved the final manuscript. 


\section{Ethics approval and consent to participate}

The Health Canada Research Ethics Board gave approval for the CHMS. All participants gave written informed consent; parents or guardians consented on behalf of children aged 6 to 13 years, and the child provided their assent to participate. The current project was approved by the IWK Health Centre Research Ethics Board, Halifax, NS, Canada (File \# 1014413).

\section{Consent for publication}

Not applicable.

\section{Competing interests}

The authors declare that they have no competing interests.

\section{Publisher's Note}

Springer Nature remains neutral with regard to jurisdictional claims in published maps and institutional affiliations.

\section{Author details}

'Departments of Pediatrics and Obstetrics \& Gynaecology, Dalhousie University, Halifax, NS, Canada. ${ }^{2}$ Department of Mathematics and Statistics, Dalhousie University, Halifax, NS, Canada.

Received: 19 April 2018 Accepted: 20 September 2018

Published online: 28 September 2018

\section{References}

1. World Health Organization. Cardiovascular diseases (CVDs). Fact sheet No. 317. Geneva: World Health Organization; 2017.

2. Linton MF, Yancey PG, Davies SS, Jerome WGJ, Linton EF, Vickers KC. The role of lipids and lipoproteins in atherosclerosis. In: De Groot LJ, Chrousos G, Dungan K, Feingold KR, Grossman A, Hershman JM, et al., editors. Endotext. South Dartmouth: MDText.com, Inc:; 2000

3. Thomas JE, Foody JM. The pathophysiology of cardiovascular disease in diabetes mellitus and the future of therapy. J Cardiometab Syndr. 2007;2: 108-13.

4. Nadeau KJ, Anderson BJ, Berg EG, Chiang JL, Chou H, Copeland KC, et al. Youth-onset type 2 diabetes consensus report: current status, challenges, and priorities. Diab Care. 2016;39:1635-42.

5. Wallace TM, Levy JC, Matthews DR. Use and abuse of HOMA modeling. Diab Care. 2004;27:1487-95.

6. Rohlfing $C L$, Little RR, Wiedmeyer HM, England JD, Madsen R, Harris MI, et al. Use of GHb (HbA1c) in screening for undiagnosed diabetes in the U.S. population. Diab Care. 2000;23:187-91.

7. American Diabetes Association. Consensus Development Conference on Insulin Resistance. 5-6 November 1997. American Diabetes Association. Diab Care. 1998;21:310-4.

8. Ehehalt S, Wiegand S, Körner A, Schweizer R, Liesenkötter KP, Partsch CJ, et al. Diabetes screening in overweight and obese children and adolescents: choosing the right test. Eur J Pediatr. 2017;176:89-97.

9. Skinner AC, Steiner MJ, Chung AE, Perrin EM. Cholesterol curves to identify population norms by age and sex in healthy weight children. Clin Pediatr (Phila). 2012;51:233-7.

10. Dathan-Stumpf A, Vogel M, Hiemisch A, Thiery J, Burkhardt R, Kratzsch J, et al. Pediatric reference data of serum lipids and prevalence of dyslipidemia: results from a population-based cohort in Germany. Clin Biochem. 2016;49:740-9.

11. Cook S, Auinger P, Huang $T T$. Growth curves for cardio-metabolic risk factors in children and adolescents. J Pediatr. 2009;155:S6.e15-26.

12. Peplies J, Jiménez-Pavón D, Savva SC, Buck C, Günther K, Fraterman A, et al. Percentiles of fasting serum insulin, glucose, HbA1c and HOMA-IR in prepubertal normal weight European children from the IDEFICS cohort. Int J Obes. 2014;38(Suppl 2):S39-47.

13. Mellerio H, Alberti C, Druet C, Capelier F, Mercat I, Josserand E, et al. Novel modeling of reference values of cardiovascular risk factors in children aged 7 to 20 years. Pediatrics. 2012;129:e1020-9.

14. Rödöö P, Ridefelt P, Aldrimer M, Niklasson F, Gustafsson J, Hellberg D. Population-based pediatric reference intervals for $\mathrm{HbA1c}$, bilirubin, albumin, CRP, myoglobin and serum enzymes. Scand I Clin Lab Invest. 2013;73:361-7.

15. Statistics Canada. Canadian health measures survey (CHMS) data user guide: cycle 1. Ottawa: Statistics Canada; 2011.
16. Statistics Canada. Canadian health measures survey (CHMS) data user guide: cycle 2. Ottawa: Statistics Canada; 2013.

17. Statistics Canada. Canadian health measures survey (CHMS) data user guide: cycle 3. Ottawa: Statistics Canada; 2015.

18. Statistics Canada. Canadian Health Measures Survey (CHMS): Instructions for combining cycle 1 and cycle 2 data. Otttawa; 2013.

19. Matthews DR, Hosker JP, Rudenski AS, Naylor BA, Treacher DF, Turner RC. Homeostasis model assessment: insulin resistance and beta-cell function from fasting plasma glucose and insulin concentrations in man. Diabetologia. 1985;28:412-9.

20. Cole TJ, Green PJ. Smoothing reference centile curves: the LMS method and penalized likelihood. Stat Med. 1992;11:1305-19.

21. Kuhle S, Maguire B, Ata N, Hamilton D. Percentile curves for anthropometric measures for Canadian children and youth. PLoS One. 2015;10:e0132891.

22. van Buuren S, Fredriks M. Worm plot: a simple diagnostic device for modelling growth reference curves. Stat Med. 2001;20:1259-77.

23. R Core Team. R: A language and environment for statistical computing. Vienna: R Foundation for Statistical Computing; 2016.

24. Rigby RA, Stasinopoulos DM. Using the box-cox t distribution in GAMLSS to model skewness and kurtosis. Stat Modelling. 2006;6:209-29.

25. Loh TP, Metz MP. Trends and physiology of common serum biochemistries in children aged 0-18 years. Pathology. 2015;47:452-61.

26. Goran Ml, Gower BA. Longitudinal study on pubertal insulin resistance. Diabetes. 2001;50:2444-50

27. Ford ES, Li C, Imperatore G, Cook S. Age, sex, and ethnic variations in serum insulin concentrations among U.S. youth: findings from the National Health and nutrition examination survey 1999-2002. Diab Care. 2006;29:2605-11.

28. Savage MO, Smith CP, Dunger DB, Gale EA, Holly JM, Preece MA. Insulin and growth factors adaptation to normal puberty. Horm Res. 1992;37(Suppl 3):70-3.

29. Ball GD, Huang TT, Gower BA, Cruz ML, Shaibi GQ, Weigensberg MJ, et al. Longitudinal changes in insulin sensitivity, insulin secretion, and beta-cell function during puberty. J Pediatr. 2006;148:16-22.

30. Robbins DC, Andersen L, Bowsher R, Chance R, Dinesen B, Frank B, et al. Report of the American Diabetes Association's task force on standardization of the insulin assay. Diabetes. 1996;45:242-56.

31. Schwartz B, Jacobs DR, Moran A, Steinberger J, Hong CP, Sinaiko AR. Measurement of insulin sensitivity in children: comparison between the euglycemic-hyperinsulinemic clamp and surrogate measures. Diab Care. 2008;31:783-8.

32. Fox C, Bernardino L, Cochran J, Essig M, Bridges KG. Inappropriate use of homeostasis model assessment cutoff values for diagnosing insulin resistance in pediatric studies. J Am Osteopath Assoc. 2017;117:689-96.

33. Diabetes Control and Complications Trial Research Group, Nathan DM, Genuth S, Lachin J, Cleary P, Crofford O, et al. The effect of intensive treatment of diabetes on the development and progression of long-term complications in insulin-dependent diabetes mellitus. N Engl J Med. 1993; 329:977-86

34. Saaddine JB, Fagot-Campagna A, Rolka D, Narayan KM, Geiss L, Eberhardt M, et al. Distribution of $\mathrm{HbA}(1 \mathrm{c})$ levels for children and young adults in the $\mathrm{U}$. S.: third National Health and nutrition examination survey. Diab Care. 2002; 25:1326-30.

\section{Ready to submit your research? Choose BMC and benefit from:}

- fast, convenient online submission

- thorough peer review by experienced researchers in your field

- rapid publication on acceptance

- support for research data, including large and complex data types

- gold Open Access which fosters wider collaboration and increased citations

- maximum visibility for your research: over $100 \mathrm{M}$ website views per year

At BMC, research is always in progress.

Learn more biomedcentral.com/submissions 